\title{
Breeding systems and continuing evolution in the endemic Sorbus taxa on Arran
}

\author{
A Robertson ${ }^{1,2}$, AC Newton ${ }^{1,3}$ and RA Ennos ${ }^{1}$ \\ ${ }^{1}$ Institute of Ecology and Resource Management, University of Edinburgh, King's Buildings, Mayfield Rd, Edinburgh EH9 3JU, Scotland
}

The Arran whitebeams Sorbus arranensis and S. pseudofennica are two endemic woody plant taxa that have evolved on Arran through hybridisation. $S$. arranensis is a triploid hybrid between the widespread diploid $S$. aucuparia and the rare tetraploid $S$. rupicola. S. pseudofennica is a tetraploid formed by crossing between $S$. arranensis and $S$. aucuparia. In order to determine the mating systems of the two endemic species six maternal trees of each taxon together with 10-12 of their seed offspring were scored for their phenotype at three microsatellite loci and one nuclear intron locus. All seeds of $S$. arranensis were identical in phenotype to their maternal parents. In S. pseudofennica, $17.5 \%$ of all seeds differed in marker phenotype from their maternal parent. The proportion of seed with nonmaternal phenotypes varied significantly among maternal trees of $S$. pseudofennica. The results suggest that the triploid $S$. arranensis is an obligate apomict, whereas the tetraploid $S$. pseudofennica is a facultative apomict. Molecular marker analysis of three trees from Arran with an unusual leaf morphology indicates that they are the product of sexual reproduction by $S$. pseudofennica, and may originate from hybridisation with $S$. aucuparia. This research demonstrates that the Sorbus taxa on Arran are participants in an active evolutionary process generating novel biodiversity. Conservation programmes for these taxa should aim to preserve this evolutionary process rather than the individual taxonomic entities that it produces. Heredity (2004) 93, 487-495. doi:10.1038/sj.hdy.6800528 Published online 14 July 2004

Keywords: breeding system; apomixis; molecular markers; Sorbus arranensis; S. pseudofennica; conservation

\section{Introduction}

Endemic plant species are rare in the British flora, and are often the product of relatively recent evolutionary events that have taken place since the last glaciation (Hollingsworth, 2003). The mode of evolution and breeding systems of these taxa influence the amount and arrangement of the genetic diversity that they currently contain, and their potential to generate new genotypic variation in the future. Understanding both the evolutionary origins and breeding systems of these endemic taxa is essential for devising appropriate programmes to conserve their present genetic variability and to maximise their potential for continued evolution, particularly in response to environmental change.

These genetic considerations are particularly relevant to the conservation of two endemic tree species, Sorbus arranensis Hedl. (Arran whitebeam) and S. pseudofennica EF Warburg (Arran service-tree), whose distributions are confined to a small number of glens in the north of Arran (Landsborough, 1897; Hedlund, 1901; Warburg, 1938). The total population size of each species is less than 500, making these the most threatened native woody taxa in

Correspondence: RA Ennos, School of Biological Sciences, University of Edinburgh, King's Buildings, Mayfield Rd, Edinburgh EH9 3JU, Scotland. E-mail: rennos@ed.ac.uk

${ }^{2}$ Current address: Department of Plant Sciences, University of Oxford, South Parks Rd, Oxford OX1 3RB, UK.

${ }^{3}$ Current address: School of Conservation Sciences, Bournemouth University, Talbot Campus, Poole, Dorset BH12 5BB, UK.

Received 23 July 2003; accepted 24 January 2004; published online 14 July 2004
Scotland (Bignal, 1980; Boyd et al, 1988). Current genetic variation within these species, and their potential to generate new genotypic variation, are expected to be low as a consequence both of their evolutionary origin and their breeding systems.

Molecular marker studies have recently confirmed that both endemic Sorbus taxa are of hybrid origin (NelsonJones et al, 2002; Robertson et al, 2004). S. arranensis $(2 n=3 x=51)$ is a triploid hybrid (chromosome constitution $\mathrm{AAB}$ ) formed between the widespread diploid species $S$. aucuparia L. (rowan) $(2 n=2 x=34)(\mathrm{BB})$ and the autotetraploid S. rupicola (Syme) Hedl. (rock whitebeam) $(2 n=4 x=68)$ (AAAA) (Hull and Smart, 1984; McAllister, 1986), which is now virtually extinct on Arran. Subsequent hybridisation of an unreduced gamete of $S$. arranensis with $S$. aucuparia has given rise to the allotetraploid S. pseudofennica $(2 n=4 x=68)$ (AABB) (Robertson et al, 2004).

In addition to their local hybrid origins, the endemic Sorbus species on Arran are believed to possess unusual breeding systems. Observations on the embryology of analogous taxa in Scandinavia suggest that both $S$. arranensis and $S$. pseudofennica are gametophytic, aposporous apomicts (Liljefors, 1953). During female reproduction in these taxa the meiotic embryo mother cell degenerates and is not involved in seed formation. Instead, an associated somatic cell develops parthenogenetically into an embryo that is genetically identical to the maternal parent (Asker and Jerling, 1992). Development of embryos is dependent on pollination, although the pollen is not involved in fertilisation, a situation known as pseudogamy (Liljefors, 1953). In terms of male 
reproduction, the tetraploid analogue of $S$. pseudofennica in Scandinavia shows high pollen fertility, whereas the triploid taxa analogous to $S$. arranensis produce no fertile pollen (Liljefors, 1955).

Both the hybrid mode of origin and the putative apomictic breeding systems of the two endemic Sorbus taxa are expected to restrict their current genetic diversity and their ability to evolve and generate novel genotypic variation in the future (Gornall, 1999) The extent of genetic variation within a taxon will initially be governed by the number of hybridisation events that have given rise to that taxon. The ability of the taxon to recombine this genetic variation, generate novel genotypes and sustain continued evolution will depend on the nature (obligate or facultative) of its breeding system.

If the endemic taxon is an obligate apomict, each independent hybridisation event giving rise to the taxon will generate a genetically distinct clone. Increases in genotypic diversity beyond the value achieved by multiple hybrid origins will be negligible in the short term, being confined to accumulation of mutations within these clones. If there is pollen fertility in the obligate apomict this will provide the opportunity for generation of new polyploid and hybrid taxa through pollination of unreduced ovules or crossing with related sexual species. However, it will not allow the generation of new genotypic variation at the same ploidy level within the existing apomictic taxon because no reduced ovules are available to be fertilised.

If the endemic taxon is a facultative apomict, initial genotypic diversity will again be dependent on the number of hybridisation events producing genetically distinct clones. However, subsequent sexual reproduction involving the fraction of ovules that are produced following normal meiosis will lead to the generation of increased genotypic variation through both segregation and recombination (Bayer et al, 1990). The greater the fraction of sexual reproduction, the greater will be the genotypic diversity generated. It should be remembered in this context that sexual reproduction and the generation of increased genotypic diversity within the taxon can only occur if the facultative apomict is capable of producing fertile pollen.

These arguments indicate that in order to assess the ability of the endemic Sorbus taxa to generate novel genetic diversity it is necessary to determine whether they behave in natural populations as obligate or facultative apomicts. A recent molecular marker analysis of population genetic structure on Arran indicates that both $S$. arranensis and S. pseudofennica comprise a small number of clones (minimum three and eight, respectively) as would be anticipated for recently derived apomictic taxa (Robertson et al, 2004). Clonal diversity within both taxa appears to have been generated principally by independent hybridisation events.

However, two of the $S$. pseudofennica individuals that were analysed in this study lacked alleles that were present in all the sampled individuals of its parent S. arranensis (Robertson et al, 2004). This suggests the possibility that additional genotypic variation has been generated in S. pseudofennica subsequent to the initial hybridisation events that gave rise to the taxon. A potential explanation for this observation is that $P$. pseudofennica is a facultative, rather than an obligate, apomict, and is capable of producing a proportion of its offspring by sexual reproduction.

The objective of this research, therefore, is to investigate the breeding systems of the endemic Sorbus taxa on Arran. We are interested in whether there is genetic evidence for apomictic reproduction of the taxa, and if so whether the apomixis is obligate or facultative. To achieve this objective, two approaches have been taken. In the first, the molecular marker phenotypes of maternal parents and offspring, sampled as seed, have been compared. If reproduction is apomictic the multilocus phenotypes of maternal parents and seeds should be identical. A difference in the marker phenotypes of a maternal tree and one of its seeds provides evidence for sexual rather than apomictic generation of this seed.

The weakness of this approach is that it may be possible to detect segregating individuals at the seed stage, but the fitness of these genotypes may be so low that they do not establish in natural populations. Therefore, to supplement this work a second approach was adopted. This relies on the fact that in natural populations on Arran a small number of established plants have been found that display an unusual leaf morphology (Lusby P, 1996, personal communication). This is intermediate between $S$. aucuparia (all leaflets free) and S. pseudofennica (all except two basal pairs of leaflets fused) (Figure 1). One of the explanations for the origin of these novel leaf morphotypes is that they are products of sexual segregation from $S$. pseudofennica. They happen to be detected because genes with large effects on leaf morphology can be recognised in the field when they segregate.

To test this suggestion these novel leaf morphotypes have been scored for their phenotype at molecular marker loci used in the present study and in that of Robertson et al (2004). Their molecular phenotype has been compared both with that of the two endemic Sorbus taxa, and with the natural seed progenies derived from these taxa. The comparisons are used to determine whether the novel morphotypes represent sexual segregants from $S$. pseudofennica that have successfully established in the natural populations on Arran.

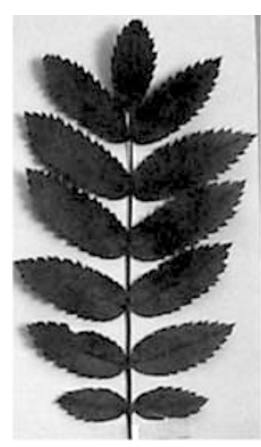

S. aucuparia

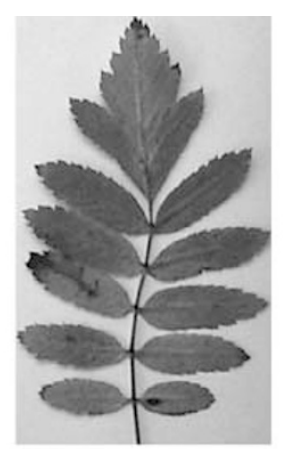

novel leaf morphotype

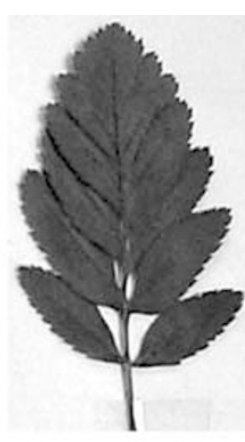

S. pseudofennica
Figure 1 Leaf morphology for Sorbus aucuparia, novel leaf morphotype of Sorbus, and S. pseudofennica. The leaves of S. aucuparia are compound pinnate. In $S$. pseudofennica all leaflets except the two lower pairs are fused. In the novel morphotype the terminal and upper pair of leaflets are fused. 


\section{Methods}

\section{Sample collection}

Leaf samples and approximately 15 mature berries were collected from six trees of $S$. arranensis and six trees of S. pseudofennica located in natural populations on Arran. Sampling sites and identity codes of the trees are given in Table 1. Within each site, sample trees were chosen at random. Seeds were removed from each fruit and stored at $4^{\circ} \mathrm{C}$. Leaves were stored frozen at $-20^{\circ} \mathrm{C}$.

Leaf samples were also collected from the three trees, situated in two populations, which display the novel leaf phenotype (Figure 1). The locations and identity codes of these trees are included in Table 1. Fresh leaf samples were used for isozyme analysis. Leaves for DNA analysis were stored frozen at $-20^{\circ} \mathrm{C}$.

\section{DNA extraction}

DNA was extracted from frozen leaves as described in Robertson et al (2004). Seeds were soaked overnight in sterile distilled water prior to DNA extraction. The embryo was then carefully separated from the endosperm under the dissecting microscope. DNA was extracted from individual embryos using a Scotlab Phytopure Plant DNA extraction kit according to the manufacturer's instructions.

\section{Genetic marker analysis}

Analysis of natural progenies: All maternal trees of both S. arranensis and S. pseudofennica, together with 10 to 12 of their progeny were assessed for variation at three nuclear microsatellite loci and a nuclear Rubisco intron locus, $r b c S$. Two of the microsatellite markers, CH02D11 (hereafter D11) and CH01H10 (hereafter H10), had been developed for Malus (Gianfranceschi et al, 1998). The third, 1204-5, had been developed for Sorbus (Robertson, 2000). Markers were selected because they had detected variation within and between the endemic Sorbus taxa on Arran in a previous study (Robertson et al, 2004).

Table 2 gives the nucleotide sequences of the primer pairs used to amplify the loci. Detailed protocols for PCR reactions and methods for gel resolution of PCR products are given in Robertson et al (2004). Owing to the polyploid nature of the taxa, analysis of the genetic marker variation was conducted on the basis of gelbanding phenotype, rather than using a strict genetic interpretation. However, for microsatellite loci individual bands are assumed to represent the products of particular alleles at the locus concerned.

Analysis of novel leaf morphotypes: All novel leaf morphotypes were scored for their phenotype at the rbcS, D11, H10 and 1204-5 loci using the methods described above. In addition, they were assayed for their phenotype at two isozyme loci (AAT and 6PGD) and a further nuclear microsatellite locus $\mathrm{CH} 02 \mathrm{~B} 3 \mathrm{~b}$ (hereafter 3B) (Gianfranceschi et al, 1998) that had been used in a previous study of genetic diversity in adult trees (Robertson et al, 2004). Table 2 gives the nucleotide sequence of primers used for amplifying locus 3B. Detailed protocols for the isozyme and microsatellite analyses are given in Robertson et al (2004).

\section{Results}

\section{Comparison of maternal parents and progeny}

$S$. arranensis: The six maternal trees of $S$. arranensis AM1-AM6 were identical to one another in banding pattern at each of the four marker loci scored. A summary of the multilocus phenotypes for the six

Table 1 Location on Arran of families of S. arranensis (AM1-AM6) and S. pseudofennica (PM1-PM6) sampled for estimating breeding system. Also shown are the locations of the three trees, $\mathrm{N}_{1}-\mathrm{N}_{3}$, with a novel leaf morphotype (Figure 1)

\begin{tabular}{lccc}
\hline Site and OS grid reference & S. arranensis families sampled & S. pseudofennica families sampled & Novel leaf morphotype sampled \\
\hline Diomhan Burn NR 925467 & AM1-AM3 & PM1-PM6 & - \\
Allt Easan Boirach NR 953474 & AM4-AM5 & - & - \\
Allt Easan Boirach NR 945496 & AM6 & - & - \\
Allt nan Calman NR 918454 & - & - & $\mathrm{N}_{1}$ \\
Catacol Burn NR 917457 & - & $\mathrm{N}_{2}, \mathrm{~N}_{3}$ \\
\hline
\end{tabular}

Table 2 Nucleotide sequences of nuclear DNA primers used in this study

\begin{tabular}{|c|c|c|}
\hline Locus & Primers $\left(5^{\prime}-3^{\prime}\right)$ & Repeat type \\
\hline $\mathrm{CH}_{02 \mathrm{D} 11^{\mathrm{a}}}$ & $\begin{array}{l}\text { Forward AGCGTCCAGAGCAACAGC } \\
\text { Reverse AACAAAAGCAGATCCGTTGC }\end{array}$ & $(\mathrm{AG})_{21}$ \\
\hline $\mathrm{CH} 02 \mathrm{~B} 03 \mathrm{~b}^{\mathrm{a}}$ & $\begin{array}{l}\text { Forward ATAAGGATACAAAAACCCTACACAG } \\
\text { Reverse GACATGTTTGGTTGAAAACTTG }\end{array}$ & $(\mathrm{GA})_{22}$ \\
\hline $\mathrm{CH} 01 \mathrm{H} 10^{\mathrm{a}}$ & $\begin{array}{l}\text { Forward TGCAAAGATAGGTAGATATATGCCA } \\
\text { Reverse AGGAGGGATTGTTTGTGCAC }\end{array}$ & $(\mathrm{AG})_{21}$ \\
\hline $1204-5^{\mathrm{b}}$ & $\begin{array}{l}\text { Forward CGGCTCTCTTCTCATCTTGG } \\
\text { Reverse TGTTAGTATGTCAGCCCAAGC }\end{array}$ & $(\mathrm{GCA})_{7}$ \\
\hline$R b c S^{c}$ & $\begin{array}{l}\text { Forward CGGCTCTCTTCTCATCTTGG } \\
\text { Reverse GTTGTCGAATCCGATGAT }\end{array}$ & \\
\hline
\end{tabular}

${ }^{a}$ Microsatellite primers derived from Malus $\times$ domestica DNA (Gianfranceschi et al, 1998).

bMicrosatellite primers derived from Sorbus DNA (Robertson, 2000).

'Nuclear Rubisco intron primers (Robertson, 2000). 
maternal trees of $S$. arranensis is given in Table 3 . When the 60 offspring of $S$. arranensis were scored they all showed banding patterns identical to those of their parents at each of the four loci (Table 3).

S. pseudofennica: Table 4 summarises the multilocus banding phenotypes of the six maternal trees of $S$. pseudofennica PM1-PM6 together with those of their families of offspring. Individual banding phenotypes for the three loci (D11, H10 and $r b c S$ ) showing variation are illustrated in Figures 2-6. Five of the six maternal parents share the same banding patterns at all loci scored. Maternal genotype PM5 differs in the possession of an additional band of intermediate mobility at the $r b c S$ locus (phenotype D, Figure 6).

In three of the S. pseudofennica families (PM1, PM2, PM4) there are no differences in the banding phenotypes between maternal individuals and their offspring at any loci. However, in the remaining three families a proportion of the offspring differ in banding patterns from their parents (Table 4, Figures 2-6). The proportion of offspring with different phenotypes ranges from 3/11 for families PM3 and PM5, to 5/10 for family PM6. Within the total seed sample, the proportion of seed with a phenotype that differs from that of its parent is 0.175 . Variation in the proportion of nonmaternal phenotypes detected in the six families of S. pseudofennica is highly significant $(P<0.005$, Fisher's exact test; Raymond and Rousset, 2002). A total of nine multilocus genotypes were detected in the seed population of S. pseudofennica.

Differences in banding pattern between maternal parents and offspring are almost entirely due to loss of bands in the offspring (19 out of 20 cases). However, in one case (family PM3, offspring 6, locus H10, phenotype C) the offspring possesses the same number of bands as the parent but one of these bands differs in its size (Figure 3). Another important feature of these results is that changes in banding pattern at one locus in an individual tend to be associated with changes at another locus. Thus, for the three families showing offspring variation there is a highly significant positive association

Table 3 Summary of molecular marker phenotypes of Sorbus arranensis maternal parents and their offspring sampled as seed in families AM1-AM6

\begin{tabular}{lllllllllll}
\hline Family & \multicolumn{3}{c}{ Phenotype at locus } & Family & \multicolumn{5}{c}{ Phenotype at locus } \\
Sample & D11 & H10 & $1204-5$ & rbc & Sample & D11 & H10 & $1204-5$ & RbcS \\
\hline AM1 & & & & & AM2 & & & & \\
Ma & A & A & A & C & Ma & A & A & A & C \\
S1-S10 & A & A & A & C & S1-S10 & A & A & A & C \\
AM3 & & & & & AM4 & & & & \\
Ma & A & A & A & C & Ma & A & A & A & C \\
S1-S10 & A & A & A & C & S1-S10 & A & A & A & C \\
AM5 & & & & & & & & & \\
Ma & A & A & A & C & Ma & A & A & A & C \\
S1-S10 & A & A & A & C & S1-S10 & A & A & A & C \\
\hline
\end{tabular}

D11 = microsatellite locus $\mathrm{CH} 02 \mathrm{D} 11, \mathrm{H} 10=$ microsatellite locus $\mathrm{CH} 01 \mathrm{H} 10,1204-5=$ microsatellite locus $1204-5, r b c S=$ rubisco intron locus. $\mathrm{Ma}=$ maternal parent, $\mathrm{S} 1, \mathrm{~S} 2$ etc. $=$ seeds. Phenotypic variants at each locus are illustrated in Figures 3, 6, 8 and 9 of Robertson et al (2004).
Table 4 Summary of molecular marker phenotypes of Sorbus pseudofennica maternal parents and their offspring sampled as seed in families PM1-PM6

\begin{tabular}{llllllllllll}
\hline Family & \multicolumn{3}{c}{ Phenotype at locus } & & Family & \multicolumn{3}{c}{ Phenotype at locus } \\
Sample & D11 & H10 & 1204-5 & rbcS & Sample & D11 & H10 & 1204-5 & rbcS \\
\hline PM1 & & & & & PM2 & & & & \\
Ma & B & B & A & C & Ma & B & B & A & C \\
S1 & B & B & A & C & S1 & B & B & A & C \\
S2 & B & B & A & C & S2 & B & B & A & C \\
S3 & B & B & A & C & S3 & B & B & A & C \\
S4 & B & B & A & C & S4 & B & B & A & C \\
S5 & B & B & A & C & S5 & B & B & A & C \\
S6 & B & B & A & C & S6 & B & B & A & C \\
S7 & B & B & A & C & S7 & B & B & A & C \\
S8 & B & B & A & C & S8 & B & B & A & C \\
S9 & B & B & A & C & S9 & B & B & A & C \\
S10 & B & B & A & C & S10 & B & B & A & C \\
S11 & B & B & A & C & & & & &
\end{tabular}

$\begin{array}{lccccccccc}\text { PM3 } & & & & & P M 4 & & & & \\ \text { Ma } & \text { B } & \text { B } & \text { A } & \text { C } & \text { Ma } & \text { B } & \text { B } & \text { A } & \text { C } \\ \text { S1 } & \text { B } & \text { B } & \text { A } & \text { C } & \text { S1 } & \text { B } & \text { B } & \text { A } & \text { C } \\ \text { S2 } & \text { C } & \text { E* } & \text { A } & \text { C } & \text { S2 } & \text { B } & \text { B } & \text { A } & \text { C } \\ \text { S3 } & \text { B } & \text { B } & \text { A } & \text { C } & \text { S3 } & \text { B } & \text { B } & \text { A } & \text { C } \\ \text { S4 } & \text { B } & \text { B } & \text { A } & \text { C } & \text { S4 } & \text { B } & \text { B } & \text { A } & \text { C } \\ \text { S5 } & \text { B } & \text { B } & \text { A } & \text { C } & \text { S5 } & \text { B } & \text { B } & \text { A } & \text { C } \\ \text { S6 } & \text { D* } & \text { C* } & \text { A } & \text { C } & \text { S6 } & \text { B } & \text { B } & \text { A } & \text { C } \\ \text { S7 } & \text { B } & \text { B } & \text { A } & \text { C } & \text { S7 } & \text { B } & \text { B } & \text { A } & \text { C } \\ \text { S8 } & \text { E* } & \text { E* } & \text { A } & \text { C } & \text { S8 } & \text { B } & \text { B } & \text { A } & \text { C } \\ \text { S9 } & \text { B } & \text { B } & \text { A } & \text { C } & \text { S9 } & \text { B } & \text { B } & \text { A } & \text { C } \\ \text { S10 } & \text { B } & \text { B } & \text { A } & \text { C } & \text { S10 } & \text { B } & \text { B } & \text { A } & \text { C } \\ \text { S11 } & \text { B } & \text { B } & \text { A } & \text { C } & & & & & \end{array}$

\begin{tabular}{|c|c|c|c|c|c|c|c|c|c|}
\hline PM5 & & & & & PM6 & & & & \\
\hline Мa & B & B & A & D & Ma & B & B & A & C \\
\hline S1 & B & B & A & D & $\mathrm{S} 1$ & $\mathrm{~A}^{*}$ & $A^{*}$ & A & C \\
\hline S2 & B & B & A & D & S2 & $\mathrm{A}^{*}$ & $\mathrm{~A}^{*}$ & A & \\
\hline S3 & B & B & A & D & S3 & $\mathrm{A}^{*}$ & $\mathrm{~A}^{*}$ & A & \\
\hline S4 & B & B & A & D & S4 & B & B & A & \\
\hline S5 & B & B & A & D & S5 & B & B & A & \\
\hline S6 & $C^{*}$ & B & A & $E^{*}$ & S6 & B & B & A & \\
\hline S7 & B & $\mathrm{A}^{*}$ & A & D & S7 & $\mathrm{A}^{*}$ & $\mathrm{~A}^{*}$ & A & \\
\hline S8 & B & B & A & D & S8 & $\mathrm{A}^{*}$ & $A^{*}$ & A & \\
\hline S9 & B & B & A & D & S9 & B & B & A & \\
\hline S10 & B & B & A & D & S10 & B & B & A & \\
\hline S11 & B & $E^{*}$ & A & D & & & & & \\
\hline
\end{tabular}

Marker phenotypes are illustrated in Figures 2-6. D11= microsatellite locus $\mathrm{CH} 02 \mathrm{D} 11, \mathrm{H} 10=$ microsatellite locus $\mathrm{CH} 01 \mathrm{H} 10$, $1204-5=$ microsatellite locus $1204-5, r b c S=$ rubisco intron locus. $\mathrm{Ma}=$ maternal parent, $\mathrm{S} 1, \mathrm{~S} 2$ etc. $=$ seed,${ }^{*}=$ a nonmaternal phenotype.

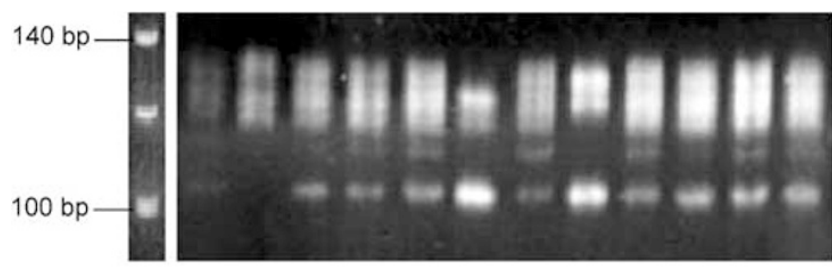

$\begin{array}{lllllllllllllllll}m & \text { S1 } & \text { S2 } & \text { S3 } & \text { S4 } & \text { S5 } & \text { S6 } & \text { S7 } & \text { S8 } & \text { S9 } & \text { S10 } & \text { S11 } & \text { Ma }\end{array}$

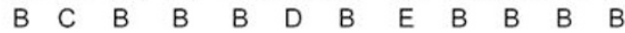

Figure 2 S. pseudofennica family PM3. Maternal and offspring banding patterns for microsatellite locus CH02D11. Letters of the alphabet designate individual phenotypes found within this family. $\mathrm{Ma}=$ maternal phenotype, S1-S11 = seed phenotypes, $m=20 \mathrm{bp}$ ladder. 


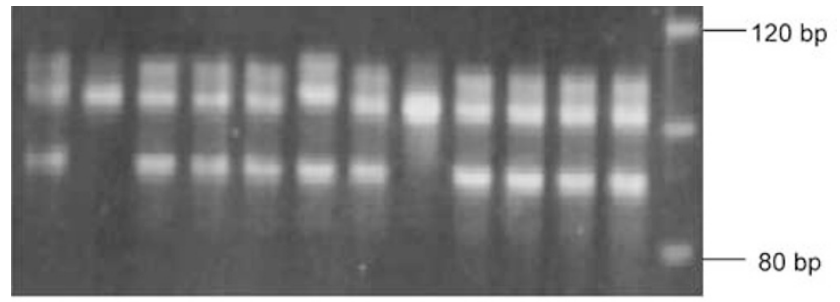

$\begin{array}{lllllllllllllllll}\mathrm{S} 1 & \mathrm{~S} 2 & \mathrm{~S} 3 & \mathrm{~S} 4 & \mathrm{~S} 5 & \mathrm{~S} 6 & \mathrm{~S} 7 & \mathrm{~S} 8 & \mathrm{~S} 9 & \mathrm{~S} 10 & \mathrm{~S} 11 & \mathrm{Ma} & \mathrm{m}\end{array}$

$\begin{array}{llllllllllll}B & E & B & B & B & C & B & E & B & B & B & B\end{array}$

Figure 3 S. pseudofennica family PM3. Maternal and offspring banding patterns for microsatellite locus $\mathrm{CH} 01 \mathrm{H} 10$. Letters of the alphabet designate individual phenotypes found within this family. $\mathrm{Ma}=$ maternal phenotype, S6-S11 = seed phenotypes, $m=20 \mathrm{bp}$ ladder. Phenotype of S6 differs from phenotype of maternal parent in length of larger band.

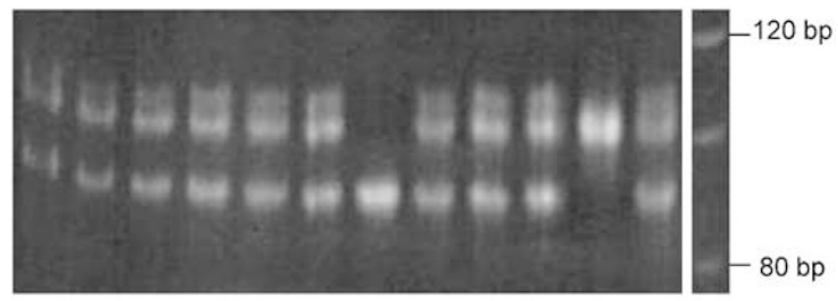

$\begin{array}{llllllllllllllllll}\mathrm{S} 1 & \mathrm{~S} 2 & \mathrm{~S} 3 & \mathrm{~S} 4 & \mathrm{~S} 5 & \mathrm{~S} 6 & \mathrm{~S} 7 & \mathrm{~S} 8 & \mathrm{~S} 9 & \mathrm{~S} 10 & \mathrm{~S} 11 & \mathrm{Ma} & \mathrm{m}\end{array}$

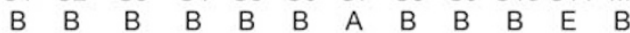

Figure 4 S. pseudofennica family PM5. Maternal and offspring banding patterns for microsatellite locus $\mathrm{CH} 01 \mathrm{H} 10$. Letters of the alphabet designate individual phenotypes found within this family. $\mathrm{Ma}=$ maternal phenotype, $\mathrm{S} 1-\mathrm{S} 11=$ seed phenotypes, $m=20 \mathrm{bp}$ ladder.

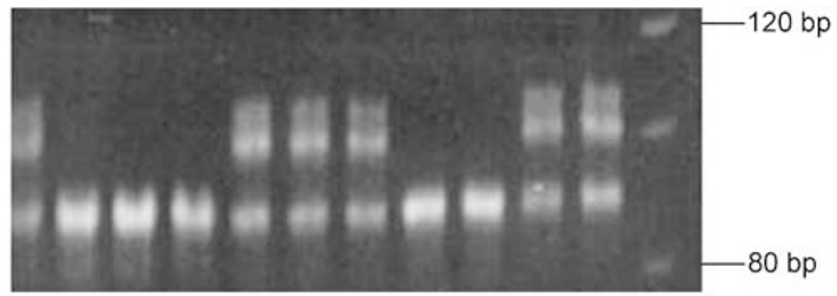

$\begin{array}{lllllllllll}M a & \text { S1 } & \text { S2 } & \text { S3 } & \text { S4 } & \text { S5 } & \text { S6 } & \text { S7 } & \text { S8 } & \text { S9 } & \text { S10 }\end{array}$

B A $\begin{array}{lllllllll}\text { A } & \text { A } & \text { B } & \text { B } & \text { B } & \text { A } & \text { A } & \text { B } & \text { B }\end{array}$

Figure 5 S. pseudofennica family PM6. Maternal and offspring banding patterns for microsatellite locus $\mathrm{CH} 01 \mathrm{H} 10$. Letters of the alphabet designate individual phenotypes found within this family. $\mathrm{Ma}=$ maternal phenotype, $\mathrm{S} 1-\mathrm{S} 10=$ seed phenotypes, $m=20 \mathrm{bp}$ ladder.

between changes occurring at the D11 and the H10 loci $(P<0.001$, Fisher's exact test; Raymond and Rousset, 2002).

Analysis of trees with novel leaf morphotype Isozyme loci: The three trees possessing the novel leaf morphotype (designated $\mathrm{N}_{1}-\mathrm{N}_{3}$ ) show the same threebanded phenotype at locus $A A T$ that is found in all

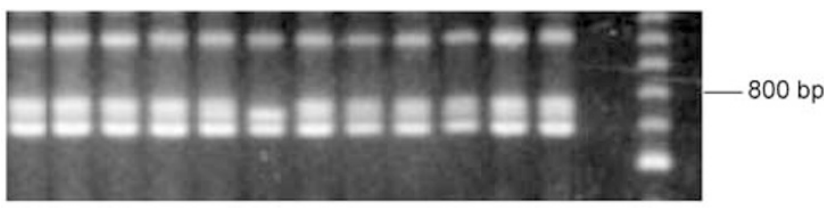

S1 S2 S3 S4 S5 S6 S7 S8 S9 S10 S11 Ma m

$\begin{array}{llllllllllll}D & D & D & D & D & E & D & D & D & D & D & D\end{array}$

Figure 6 S. pseudofennica family PM5. Maternal and offspring banding patterns for the $r b c S$ locus. Letters of the alphabet designate individual phenotypes found within this family. $\mathrm{Ma}=$ maternal phenotype, $\mathrm{S} 1-\mathrm{S} 11=$ seed phenotypes, $m=100 \mathrm{bp}$ ladder.

6PGD

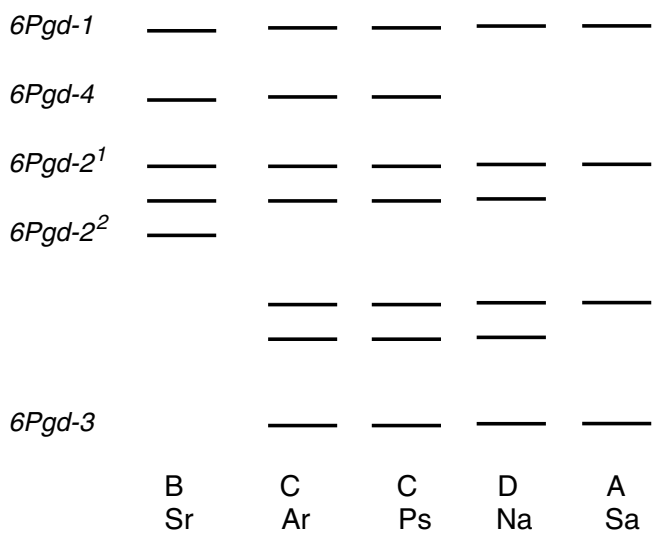

Figure 7 Isozyme phenotypes at locus 6PGD in Sorbus taxa. Letters of the alphabet designate individual phenotypes. $\mathrm{Sr}=S$. rupicola $\mathrm{Ar}=$ S. arranensis, $\mathrm{Ps}=$ S. pseudofennica, $\mathrm{Na}=$ novel leaf morphotype, $\mathrm{Sa}=S$. aucuparia

individuals of both $S$. arranensis and S. pseudofennica (Figure 2 in Robertson et al, 2004). At locus 6PGD, the phenotype of trees $\mathrm{N}_{1}-\mathrm{N}_{3}$ is similar to that in S. arranensis and S. pseudofennica, except that it lacks the $6 p g d-4$ band (Figure 7). This band originates from the S. rupicola parent (Robertson et al, 2004).

\section{Nuclear DNA markers}

Table 5 summarises the phenotypes of trees $\mathrm{N}_{1}-\mathrm{N}_{3}$ at the nuclear microsatellite and rubisco intron loci scored in this study. Also included in the table is the complete list of multilocus phenotypes detected at the same loci in a population survey of $S$. arranensis and S. pseudofennica trees on Arran (Robertson et al, 2004).

Trees $\mathrm{N}_{1}$ and $\mathrm{N}_{3}$, sampled from different populations (Table 1), show an identical multilocus, molecular phenotype (phenotype N1). This suggests that they are members of the same apomictic clone. This phenotype is different from that of tree $\mathrm{N}_{2}$ (phenotype N2). Neither N1 nor N2 was detected in the widespread survey of S. arranensis or S. pseudofenica on Arran. The trees with the novel leaf morphology show molecular phenotypes $\mathrm{G}$ and $\mathrm{F}$ at the microsatellite marker locus D11, and phenotypes $\mathrm{E}$ and $\mathrm{F}$ at microsatellite marker locus H10, 
Table 5 Summary of multilocus genotypes present in populations of S. arranensis and S. pseudofennica on Arran and the number of each genotype scored $(n)$

\begin{tabular}{lllllllll}
\hline Taxon & Multilocus & & \multicolumn{4}{c}{ Phenotype at locus } \\
\cline { 5 - 9 } & genotype & & $\mathrm{n}$ & & II & III & IV & rbcS \\
\hline Novel leaf morphotype & $\mathrm{N} 1\left(\mathrm{~N}_{1}, \mathrm{~N}_{3}\right)$ & 2 & G & B & F & A & C \\
& $\mathrm{N} 2\left(\mathrm{~N}_{2}\right)$ & 1 & F & B & E & A & C
\end{tabular}

S. arranensis

$\begin{array}{lrlllll}\text { A1 } & 171 & \text { A } & \text { A } & \text { A } & \text { A } & \text { C } \\ \text { A2 } & 7 & \text { A } & \text { A } & \text { A } & \text { C } & \text { C } \\ \text { A3 } & 1 & \text { A } & \text { A } & \text { A } & \text { B } & \text { C }\end{array}$

S. pseudofennica

$\begin{array}{lllllll}\text { P1 } & 70 & \text { B } & \text { A } & \text { B } & \text { A } & \text { C } \\ \text { P1 } & \text { 52 } & \text { B } & \text { A } & \text { B } & \text { A } & \text { D } \\ \text { P3 } & 7 & \text { A } & \text { A } & \text { D } & \text { A } & \text { C } \\ \text { P4 } & 4 & \text { B } & \text { A } & \text { D } & \text { A } & \text { C } \\ \text { P5 } & 4 & \text { A } & \text { A } & \text { C } & \text { A } & \text { C } \\ \text { P6 } & 1 & \text { B } & \text { B } & \text { B } & \text { A } & \text { C } \\ \text { P7 } & 1 & \text { B } & \text { A } & \text { D } & \text { C } & \text { C } \\ \text { P8 } & 1 & \text { B } & \text { B } & \text { B } & \text { A } & \text { E }\end{array}$

Individuals were assessed for their phenotype at four polymorphic microsatellite loci $(\mathrm{I}=\mathrm{CH} 02 \mathrm{D} 11, \mathrm{II}=\mathrm{CH} 02 \mathrm{~B} 3 \mathrm{~b}, \mathrm{III}=\mathrm{CH} 01 \mathrm{H} 10$ and $\mathrm{IV}=1204-5)$ and one nuclear intron locus $r b c S$. Phenotypic variants at each locus are illustrated in Figures 8 and 9 (this paper) and Figures 3, 6, 7, 8 and 9 of Robertson et al (2004).

$140 \mathrm{bp}$

$100 \mathrm{bp}$
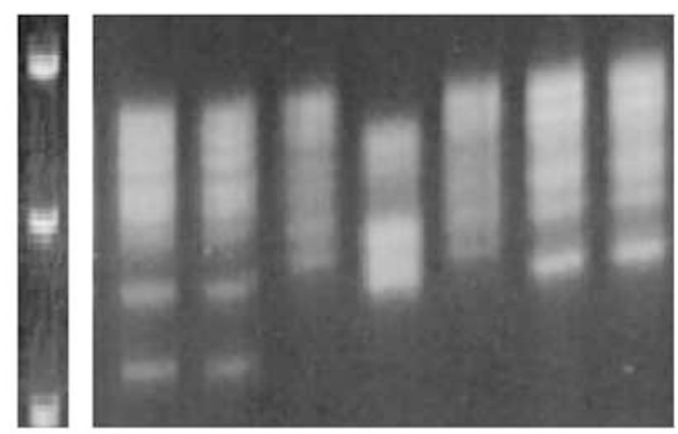

$$
m \quad \begin{array}{ccccccc}
\mathrm{PS} & \mathrm{Ps} & \mathrm{N}_{1} & \mathrm{~N}_{2} & \mathrm{~N}_{3} & \mathrm{Ar} & \mathrm{Ar} \\
\mathrm{B} & \mathrm{B} & \mathrm{G} & \mathrm{F} & \mathrm{G} & \mathrm{A} & \mathrm{A}
\end{array}
$$

Figure 8 Novel leaf morphotypes in Sorbus. Banding patterns for microsatellite locus CH02D11. Letters of the alphabet designate individual phenotypes. $\mathrm{N}_{1}-\mathrm{N}_{3}=$ novel leaf morphs, $\mathrm{Ar}=$ S. arranensis, $\mathrm{Ps}=S$. pseudofennica, $m=20 \mathrm{bp}$ ladder.

that were not present in the survey of $S$. arranensis and S. pseudofennica. Figures 8 and 9 illustrate these new banding phenotypes. They are characterised by loss of bands normally present in the endemic taxa, by new combinations of bands that had previously been detected in these taxa, and by bands not previously detected (phenotypes F and G locus D11). It is interesting to note that phenotype $\mathrm{E}$ at locus $\mathrm{H} 10$ is present in seed families PM3 and PM5 of S. pseudofennica (Table 4), but not in established trees of this taxon.

\section{Discussion}

The key observation in this study is that all offsprings of $S$. arranensis share the molecular phenotype of their

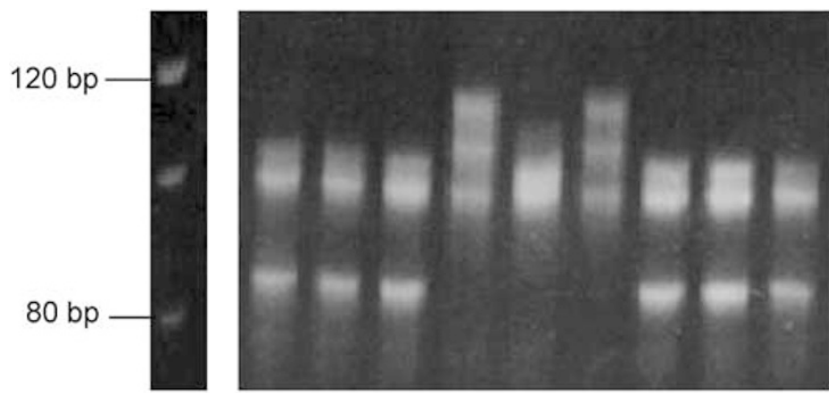

$\begin{array}{llllllllll}m & P S & P S & P S & N_{1} & N_{2} & N_{3} & P S & P S & P s\end{array}$

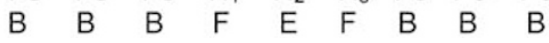

Figure 9 Novel leaf morphotypes in Sorbus. Banding patterns for microsatellite locus $\mathrm{CH} 01 \mathrm{H} 10$. Letters of the alphabet designate individual phenotypes. $\mathrm{N}_{1}-\mathrm{N}_{3}=$ novel leaf morphs, $\mathrm{Ps}=S$. pseudofennica, $m=20 \mathrm{bp}$ ladder.

maternal parent, whereas in S. pseudofennica, a significant proportion of offspring differ from their maternal parent. The differences between maternal parents and offspring in S. pseudofennica are almost entirely due to loss of bands at microsatellite loci D11 and H10. These same bands are present in S. arranensis parents, but are never missing in their offspring. We can conclude that the observed differences between the taxa cannot be ascribed to variation in the methods used to detect progeny with nonmaternal genotypes. The breeding systems of the two taxa are therefore significantly different.

There is no evidence from the data that anything other than apomictic production of seed is occurring in the triploid $S$. arranensis. However, in the tetraploid S. pseudofennica segregation of molecular markers indicates that a significant proportion of offspring are the product of sexual reproduction. It is interesting to consider how this difference in mating system might arise. Sexual reproduction in an apomictic species can be constrained by three factors; a failure to initiate sexual development of embryos, an inability to produce viable reduced female gametes, and an inability successfully to fertilise these gametes (Asker, 1979; Grossniklaus et al, 2001).

In the triploid $S$. arranensis, even if a proportion of embryo sacs follow normal sexual development, they are likely to experience difficulties in meiosis, producing nonviable ovules. Moreover, difficulties in meiosis during pollen production mean that virtually no fertile pollen is produced in triploid taxa (Liljefors, 1955). Therefore, in the triploid hybrid it is unlikely that anything other than apomictic reproduction will result in viable offspring, even if significant numbers of embryo-sacs begin normal sexual rather than aposporous development.

In the allotetraploid S. pseudofennica, on the other hand, meiosis is likely to proceed normally with the opportunity for regular pairing of chromosomes. If any sexual development of embryo-sacs is initiated it will result in viable female gametes. In terms of male gametes, there is likely to be production of fertile pollen, based on data from the allotetraploid analogue of $S$. pseudofennica in Scandinavia (Liljefors, 1955). There is, therefore, the possibility of converting those embryos that follow 
normal sexual development into sexually produced offspring.

This means that the differences in breeding systems of the triploid and tetraploid taxa are not necessarily associated with differences in the rate at which sexual embryo-sac development is initiated. It may instead be a function of the ability of the taxa to follow this development through to the production of viable sexual offspring. In the genus Rubus a situation of obligate apomixis in triploids and facultative apomixis in tetraploid taxa has been well documented (Antonius and Nybom, 1995) and is similarly explained by inability of triploid taxa to produce viable sexual seed.

Among S. pseudofennica families, the detected frequency of sexually produced offspring is significantly heterogeneous. All families are derived from maternal trees that are identical in banding phenotype at the two microsatellite loci (D11 and H10) used to estimate rates of sexual reproduction. Thus, the probability of detecting sexual progeny does not differ among families. Therefore, the observed heterogeneity indicates a real difference in rates of sexual reproduction among S. pseudofennica families.

The overall proportion of seeds detected to be the products of sexual reproduction is $17.5 \%$. It is important to ask whether this is an accurate estimate of the sexual reproduction rate. To answer this question, we note that $92 \%$ of the S. pseudofennica individuals in the population from which maternal parents and seed were sampled possess identical molecular phenotypes at the informative loci D11 and H10. These phenotypes are also possessed by all the maternal trees sampled. If these maternal trees produce sexual offspring, any self-fertilisation event and the vast majority of outcrosses will involve mating between males and female parents with identical genotypes at loci D11 and CH10.

Under either disomic or tetrasomic inheritance, sexual progeny will be detected at significant frequencies only where there is segregation of an allele present in a single copy at these loci, resulting in the loss of a band in the molecular phenotype of the progeny. All of the sexual progeny except one are recognised in this manner. Under disomic or tetrasomic inheritance, crosses between two parents possessing a single copy of the same allele will segregate such that one-quarter of their offspring will lack this allele. Thus, if loss of a particular band is used to detect a sexual cross, only a quarter of the offspring produced by a sexual cross will be recognised. However, in this study, loss/retention of six different bands at the D11 and CH10 loci has been used to detect sexually produced offspring. This considerably reduces the chance that sexual reproduction events will go undetected.

If loss of different bands at loci D11 and H10 represented independent events then the probability of detecting a sexual event would be $1-(0.75)^{6}=0.822$. The true rate of production of sexual offspring would therefore be $0.175 / 0.822=0.21$. However, loss of bands will not be independent events owing to allelism of bands at the D11 and H10 loci and possible linkage between the loci. Indeed, the observation of a significant association between changes at the D11 and H10 loci in segregating families demonstrates that losses of bands at these two loci are not independent events. In all, $21 \%$ should therefore be regarded as a minimum estimate for the overall frequency of sexual offspring produced by $S$. pseudofennica.

Although almost all differences between maternal parents and seed progeny involve loss of bands from the molecular phenotype, one seed displays a band not found in the maternal parent (offspring 6, family PM3, locus H10). This nonmaternal band is present in four out of the 79 trees of S. pseudofenncia (phenotype P5) sampled within the Diomhan Burn population (Table 5). The most likely origin for the progeny with the nonmaternal band is an outcross event in which an individual of the P5 phenotype was the pollen parent.

The allele coding for this band in phenotype P5 is likely to have been inherited from $S$. aucuparia in the hybridisation event that led to the formation of this clone of S. pseudofennica (Robertson et al, 2004). It will therefore be present in a single copy in the P5 phenotype. The expected frequency of the allele in the outcross pollen pool of the $S$. pseudofennica population is therefore 4/ $(79 \times 2)=0.0253$ and the expected frequency of sexual offspring carrying this allele is $0.0253 t$ where $t$ is the outcrossing rate for sexual offspring in S. pseudofennica. Among offspring of parents known to be reproducing sexually, the frequency of progeny with this allele is $1 / 32=0.0312$. Thus, the observed result is not inconsistent with a model in which sexual offspring of $S$. pseudofennica are produced by random outcrossing where $t=1.0$.

In summary, the analysis of molecular marker data in maternal and progeny genotype arrays indicates that $S$. arranensis produces seed exclusively by apomictic reproduction. In $S$. pseudofennica, apomixis also takes place, but a minimum proportion of 0.21 of its seeds are produced sexually, probably by random outcrossing. There is considerable heterogeneity in the frequency of sexual seed production among maternal parents of S. pseudofennica.

Analysis of molecular markers in maternal plants and progeny genotype arrays has been used on a number of previous occasions to estimate rates of sexual reproduction in facultative apomicts. Bayer et al (1990) estimated rates of sexual reproduction of 0.41 and 0.86 in two populations of the tetraploid Antennaria media that differed in the frequency of male plants. There was also evidence for heterogeneity in the rates of sexual reproduction among families. In the tetraploid grass, Paspalum notatum families varied from 4 to $100 \%$ in the frequency of sexual progeny (Ortiz et al, 1997). In contrast, uniformly low estimates of sexual reproduction $(0.5 \%)$ were detected in the savannah grass Hyparrhenia diplandra (Durand et al, 2000). Estimates of sexual reproduction rates for $S$. pseudofennica lie in the middle of the range so far recorded for facultative apomicts.

Given this very substantial rate of sexual reproduction of seeds by $S$. pseudofennica, is there any evidence that sexually produced seeds are able to establish in the population? In a previous survey of the S. pseudofennica population, two individuals were found whose molecular phenotype lacked bands at loci $3 \mathrm{~B}$ and $r b c S$ that are invariably present in S. arranensis (Robertson et al, 2004). Because S. pseudofennica originates from an unreduced gamete of $S$. arranensis it was suggested that these two individuals may represent sexually reproduced offspring 
of S. pseudofennica in which segregation had taken place at the two loci. This interpretation is supported by the detection of substantial segregation of markers following sexual reproduction by $S$. pseudofennica in the present study. Detection of segregants in the adult population implies that sexually produced offspring have been able to establish in natural populations.

To provide further evidence for the presence of sexually reproduced genotypes in natural populations three further individuals $\left(\mathrm{N}_{1}-\mathrm{N}_{3}\right)$ proposed (on the basis of their novel leaf morphology) to have been produced by sexual reproduction from $S$. pseudofennica, have been analysed for their molecular marker phenotypes. The analysis shows that trees $\mathrm{N}_{1}$ and $\mathrm{N}_{3}$ share the same multilocus phenotype, implying that they are members of the same apomictic clone. They are genetically distinct from tree $\mathrm{N}_{2}$. Neither of these two multilocus marker phenotypes has been found before in S. arranensis or in established $S$. pseudofennica trees with normal leaf morphology.

The simplest explanation for the origin of the novel phenotypes N1 and N2 is that they are tetraploid, sexual segregants from crosses between individuals of $S$. pseudofennica. In the sites where the novel morphotypes are found there are substantial numbers of $S$. pseudofennica (20 and 24) belonging to three different clones, and there is ample opportunity for sexual crossing between individuals (Robertson et al, 2004). The isozyme phenotype of the novel morphotypes could occur through segregation leading to loss of the 6PGD-4 allele. Some of the microsatellite evidence is also compatible with this hypothesis. Phenotype E at locus $\mathrm{H} 10$ in individual $\mathrm{N}_{2}$ could be produced by segregation and indeed is present among the sexually produced seed of $S$. pseudofennica. For individuals $N_{1}$ and $N_{3}$ phenotype $F$ at locus $H 10$ could be explained by inheritance of two bands known to be present in the adult population of S. pseudofennica. However, at locus D11 phenotypes F and G appear to include bands that are not present in any individuals of S. pseudofennica (or for that matter S. arranensis). This casts doubt on the hypothesis that the novel morphotypes originate from crosses within S. pseudofennica.

A further difficulty with this hypothesis concerns the generation of the novel leaf morphology. As an allotetraploid S. pseudofennica contains two genomes donated by the simple leaved $S$. rupicola (denoted AA), and two from $S$. aucuparia (BB) that possesses compound leaves. Combination of the two genomes in S. pseudofennica $(\mathrm{AABB})$ results in a compound leaf with all except the lowest two pair of leaflets fused (Figure 1). If chromosomes show disomic behaviour, as expected in an allotetraploid, then crosses within S. pseudofennica should generate individuals with the same chromosomal balance. The characteristic leaf morphology of S. pseudofennica should therefore be retained in sexual crosses within S. pseudofennica. The only way of generating a genotype with the novel leaf morphology from crosses within $S$. pseudofennica is to propose tetrasomic inheritance. This could generate tetraploid individuals inheriting three genomes from $S$. aucuparia and one from $S$. rupicola $(\mathrm{ABBB})$ to give the novel leaf morphotype.

An alternative explanation for the novel morphotypes that does not require tetrasomic inheritance is that they are the products of sexual outcrossing between $S$. pseudofennica (AABB) and S. aucuparia (BB). This would give a triploid hybrid $(\mathrm{ABB})$ with a predominance of chromosomes from $S$. aucuparia. Such a hybrid may exhibit the novel leaf morphotype. S. aucuparia and $S$. pseudofennica occur sympatrically in the sites where the novel morphotype is found, so the opportunity for such hybridisation in either direction is certainly present. This hypothesis could explain the presence of novel bands at locus D11 in phenotypes N1 and N2 if they were donated by genetically variable $S$. aucuparia.

Studies of a parallel evolutionary situation in Scandinavia support the possibility of a hybrid origin for the novel morphotype. Lindenberg recognised there a taxon with a leaf morphology intermediate between $S$. aucuparia and the analogue of S. pseudofennica (S. hybrida) (Lindenberg in Hartman, 1879, p 271). Hedlund (1901) named the taxon Sorbus meinichii (Lindeb.) Hedl. Liljefors (1953) later showed that this name had been applied to three cytological entities, a tetraploid apomict, a triploid sexual hybrid, and a triploid apomict. He proposed a new name for the triploid, Sorbus teodori Liljef. Recent morphological and cytological work has supported the proposal that $S$. teodori has resulted from a cross between S. aucuparia and S. hybrida (Bolstad and Salvesen, 1999). The origin of the novel leaf morphotype on Arran could likewise be resolved by cytological work. If the trees are the offspring of crosses within S. pseudofennica they will be tetraploid, while if they are the offspring of hybridisation with $S$. aucuparia they will be triploid.

Notwithstanding the detailed origin of the novel leaf morphotype, the molecular marker analysis presented here demonstrates that the Sorbus complex on Arran is an actively evolving entity that does not rely solely on rare hybridisation events and mutation for the generation of new genotypic diversity. Although the triploid taxon $S$. arranensis is an obligate apomict with relatively little variability, it has given rise on multiple occasions to a tetraploid taxon, S. pseudofennica, which produces a substantial proportion of its seed through sexual production. These sexually produced seeds have the ability to establish in natural populations, significantly increasing the diversity of genotypes present on Arran. There is also a suggestion that further diversity is being produced within the complex by crosses between S. pseudofennica and S. aucuparia.

These results have an important bearing on conservation policy for the endemic Sorbus species on Arran. Both endemic taxa together with the widespread species $S$. aucuparia are involved in active evolution of the polyploid complex (Soltis and Soltis, 1993). S. arranensis, although evolutionarily inert on its own, acts in concert with sexual $S$. aucuparia to generate novel genotypes of S. pseudofennica. S. pseudofennica generates further genotypic variation through sexual outcrossing and possibly hybridisation with $S$. aucuparia. To maximise the potential for evolution of this complex and the long-term survival of an endemic Sorbus entity on Arran, the conservation management objective should be to ensure that all the components of the evolutionary complex, $S$. arranensis, S. pseudofennica and S. aucuparia are retained in the northern glens of Arran. The aim must be to perpetuate the dynamic evolutionary processes that have given rise to the endemic taxa in situ, rather than statically to preserve the named taxonomic entities that have emerged from this process. 


\section{Acknowledgements}

We would like to thank Chris Sydes, Juliet Vickery, Philip Lusby and Tony Church for their enthusiasm, advice and help with the project and John Morman, Mick Carr and Nicola Preston for their technical advice and assistance. This work was supported by the Natural Environment Research Council and Scottish Natural Heritage.

\section{References}

Antonius K, Nybom H (1995). Discrimination between sexual recombination and apomixis/automixis in a Rubus plant breeding programme. Hereditas 123: 205-213.

Asker SE (1979). Progress in apomixis research. Hereditas 91: 231-240.

Asker SE, Jerling L (1992). Apomixis in Plants, CRC-Press: Boca Raton, FL.

Bayer RJ, Ritland K, Purdy BG (1990). Evidence of partial apomixis in Antennaria media (Asteraceae: Inuleae) detected by the segregation of genetic markers. Am J Bot 77: 1078-1083.

Bignal E (1980). The endemic whitebeams of north Arran. Glasgow Nat 20: 59-64.

Bolstad AM, Salvesen PH (1999). Biosystematic studies of Sorbus meinichii (Rosaceae) at Moster, S. Norway. Nordic J Bot 19: $547-559$.

Boyd WE, Laing AI, Steven G, Dickson JH (1988). The history and present management of two rare endemic trees on the Island of Arran, Scotland. Env Cons 15: 65-66.

Durand J, Garnier L, Dajoz I, Mousset S, Veuille M (2000). Gene flow in an apomictic Poacea, the savanna grass Hyparrhenia diplandra. Genetics 156: 823-831.

Gianfranceschi L, Seglias N, Tarchini R, Komjanc M, Gessler C (1998). Simple sequence repeats for genetic analysis. Theor Appl Gen 96: 1069-1076.

Gornall RJ (1999). Population genetic structure in agamospermous plants. In: Hollingsworth PM, Bateman RM, Gornall RJ (eds) Molecular Systematics and Plant Evolution, Taylor \& Francis: London. pp 118-138.
Grossniklaus U, Nogler GA, van Dijk PJ (2001). How to avoid sex. The genetic control of gametophytic apomixis. The Plant Cell 13: 1491-1498.

Hartman CJ (1879). Handbog i Skandinaviens flora, ed. 11. Stockholm.

Hedlund T (1901). Monographie der Gattung Sorbus. Kungliga Svenska Vetenskaps-Akademiens Handlingar 35: 1-147.

Hollingsworth PM (2003). Taxonomic complexity, population genetics and plant conservation in Scotland. Bot J Scotl 55 55-63.

Hull P, Smart GJB (1984). Variation in two Sorbus species endemic to the Isle of Arran, Scotland. Ann Bot 53: 641-648.

Landsborough D (1897). Pyrus aria and its varieties in Arran Trans Proc Bot Soc Edin 21: 56-62.

Liljefors A (1953). Studies on propagation, embryology and pollination in Sorbus. Acta Horti Bergiani 16: 277-329.

Liljefors A (1955). Cytological studies in Sorbus. Acta Horti Bergiani 17: 47-113.

McAllister HA (1986). The Rowan and Its Relatives (Sorbus spp.) Ness series 1. Ness Gardens, University of Liverpool Botanic Gardens: Liverpool.

Nelson-Jones EB, Briggs D, Smith AG (2002). The origin of intermediate species of the genus Sorbus. Theor Appl Genet 105: 953-963.

Ortiz JPA, Pessino SC, Leblanc O, Hayward MD, Quarin CL (1997). Genetic fingerprinting for determining the mode of reproduction of Paspalum notatum, a subtropical apomictic forage grass. Theor Appl Genet 95: 850-856.

Raymond M, Rousset F (2002) Genepop on the web. http:// wbiomed.curtin.edu.au/genepop/.

Robertson A (2000). The origin, evolution and conservation of the Arran Sorbus microspecies. PhD Thesis, University of Edinburgh.

Robertson A, Newton AC, Ennos RA (2004). Multiple hybrid origins, genetic diversity and population genetic structure of two endemic Sorbus taxa on Arran. Mol Ecol 13: 123-134.

Soltis DE, Soltis PS (1993). Molecular data and the dynamic nature of polyploidy. Crit Rev Pl Sci 12: 243-273.

Warburg EF (1938). The origin and distribution of the British forms of Sorbus. Report of the Annual Meeting, pp 507-508. British Association for the Advancement of Science. 